\title{
Improving the Isotretinoin Photostability by Incorporating in Microemulsion Matrix
}

\author{
Mrunali R. Patel, ${ }^{1}$ Rashmin B. Patel, ${ }^{2}$ Jolly R. Parikh, ${ }^{2}$ and Bharat G. Patel ${ }^{3}$ \\ ${ }^{1}$ Indukaka Ipcowala College of Pharmacy, New Vallabh Vidyanagar, Anand, Gujarat 388 121, India \\ ${ }^{2}$ A. R. College of Pharmacy and G. H. Patel Institute of Pharmacy, Vallabh Vidyanagar, Anand, Gujarat 388 120, India \\ ${ }^{3}$ Institute of Science and Technology for Advance Studies and Research, Vallabh Vidyanagar, Anand, Gujarat 388 120, India \\ Correspondence should be addressed to Mrunali R. Patel, mrunalipatel@gmail.com
}

Received 13 April 2011; Accepted 11 May 2011

Academic Editor: H. Arima

Copyright ( $\odot 2011$ Mrunali R. Patel et al. This is an open access article distributed under the Creative Commons Attribution License, which permits unrestricted use, distribution, and reproduction in any medium, provided the original work is properly cited.

The present paper demonstrates the increased photostability of isotretinoin when loaded in microemulsion. The photodegradation of isotretinoin, in methanol and microemulsion formulation was studied under direct sun light. The photodegradation process was monitored by UV spectrophotometry. In methanol solution, isotretinoin undergoes complete photodegradation just within a few minutes of light exposure. Isotretinoin incorporated in microemulsion formulation showed an increased stability in comparison to the methanol solutions. In particular for isotretinoin, a residual concentration of $75 \%$ was still present after a light irradiance versus a residual value of just $16 \%$ measured at the same time in methanol solution. Further, degradation kinetic parameters of isotretinoin-loaded microemulsion formulation were demonstrated increase isotretinoin half-life about five-times in comparison with a methanol solution under a direct sun light.

\section{Introduction}

Topical retinoids constitute the core of nearly all therapeutic programs in the mild to moderate acne with an ability to be used to treat noninflammatory and inflammatory acne. The therapeutic success in acne is highly dependent on a regular application of the topical agents over a prolonged period of time. But there are several factors which would seem to indicate that topical application may be problematic. Among them is the extremely low solubility of the compounds, limiting incorporation into an acceptable vehicle, photoinstability which may render topically applied drug ineffective and tolerability problem leading to significant erythema, dryness, peeling, scaling and irritation of skin. This results in discontinuation of treatment or compliance problems in patients who continue treatment [1-3].

Isotretinoin (ITN), a derivative of retinoic acid (13cis-retinoic acid), is the most effective compound with potential to suppress acne over the long term. However, low solubility of ITN limits its incorporation in a suitable vehicle while its poor photostability renders the topically applied drug ineffective. Therefore, the development of novel formulations characterized by high photoprotection of ITN seems to be important, as well an efficient investigation about the photodegradation processes occurring in the ITN in the developed formulations. The inclusion of the drugs in the novel formulation matrix represents an approach of growing interest to the problem of light-sensitive drugs [4-7].

Novel drug delivery strategies like liposomes, niosomes, aspasomes, microsponges, microemulsion (ME), hydrogels, \& solid-lipid nanoparticles can play a pivotal role in optimizing \& enhancing the topical delivery of antiacne agents by either modulating their physicochemical \& biopharmaceutical properties or minimizing/eliminating the side effects associated with them, thus offering better patient compliance [8-10].

The interest in the development of the drug delivery system for ITN based on ME is because they offer an interesting \& potentially a quite powerful way for drug delivery as colloidal drug carrier due to their versatility $\&$ attractive advantages. The favourable cutaneous drug delivery properties due to large concentration gradients provided by large drug solubility potential of vehicle affinity for the drug together with ease of formulation, their physical 
\& thermodynamic stability makes $\mathrm{ME}$ a very promising vehicles for future topical formulations. Also, topically applied microemulsions (MEs) have been demonstrated to significantly increase the cutaneous absorption of both lipophilic and hydrophilic drugs compared to conventional vehicles for example, aqueous solutions, neat oil phases, micellar solutions, emulsions, and liposomes [11-13].

Drugs photostability represents a significant problem in pharmaceutical research, several articles and reviews dealing with different aspects of such studies are present. Photostability testing for new drugs is included as integral part of stress testing in the ICH guideline [14] and really is an essential activity of the pharmaceutical industry [15]. Formulation and manufacturing process seem to be of decisive importance for drug photostability [16] and several approach systems have been proposed in order to enhance stability for a number of drugs [17].

Photostability studies for retinoic acid formulations have been performed on lotion [18], pharmaceuticals [19], and cosmetics [20]. The inclusion of retinoic acid in liposomes was reported to protect the drug against photodegradation [21], as well as a profound characterization of retinoic acid-liposome [22] and niosome complexes [23] has been described. Shah et al. evaluated the ability of solid-lipid nanoparticle in improving the photostability of tretinoin as compared to tretinoin in methanol and reported that encapsulation of tretinoin in solid-lipid nanoparticle resulted in a significant improvement in its photostability in comparison to methanolic solution and also prevented its isomerization [24]. Ioele et al. reported that tretinoin and ITN undergo a stepwise photodegradation in ethanol with a first very rapid isomerization to 13-cis and 9-cis isomers, respectively, both followed by a slower degradation to give several isomers. Inclusion of the drugs in liposome matrix was proved to show an improved stability to light. Also, the fast isomerization realized in ethanol from both compounds is avoided in liposomes. They were demonstrated to decrease tretinoin half-life about twelve-times in comparison with an ethanol solution, under a light power of $250 \mathrm{~W} / \mathrm{m}^{2}$. Further, authors concluded that the inclusion of ITN in liposomes showed a worse performance as compared to tretinoin in liposomes, probably due to low inclusion attributable to its nonlinear molecular structure [25].

No literature (to date) was found about the inclusion of ITN in MEs. Hence the present study was undertaken with an aim to increase the photostability of ITN when loaded in ME. ITN-loaded matrixes were subjected to photodegradation studies, either in solution or ME, and an interpretation of the photodegradation process of ITN by determination of kinetic parameters was tried as a complementary aim of the photostability study. Analytical monitoring of the retinoic acids was performed by UV spectrophotometry.

\section{Materials and Methods}

2.1. Apparatus. Absorbance was measured, and spectra were recorded over the wavelength range $200-800 \mathrm{~nm}$ in two matched quartz cells with a $1 \mathrm{~cm}$ light path using a double beam Perkin Elmer Lambda 19 (Perkin Elmer, Norwalk, CT) UV-Visible spectrophotometer.

2.2. Chemicals. ITN was procured as gratis samples from Astron Research Ltd. (Ahmedabad, India). Analytical-grade methanol (E. Merck, Mumbai, India) was used for the sample preparation. Isopropyl Myristate (IPM) was purchased form National Chemicals (Vadodara, India). Caprylocaproyl macrogol-8-glyceride and polyglyceryl oleate were procured as gratis sample form Gattefosse (Saint-Priest, France). Double distilled water was used for the preparation of MEs to avoid surface active impurities.

2.3. Laboratory Precautions. To minimize drugs photodegradation, all handling of ITN was carried out under red lamp $(60 \mathrm{~W})$ and whenever possible amberized glassware was used.

2.4. Preparation of Standard Stock Solutions. Stock solution was prepared by weighing ITN pure powder (10 mg), weighed powder was accurately transferred to a volumetric flask of $100 \mathrm{~mL}$ and dissolved in and diluted to the mark with methanol to obtain a standard stock solution of ITN $(100 \mu \mathrm{g} / \mathrm{mL})$. Working standards, to obtain concentrations within a range of $5.0-40.0 \mu \mathrm{g} / \mathrm{mL}$ for ITN, were prepared by appropriate dilution with methanol of the stock solutions and used to set up the calibration curves.

2.5. Preparation of ITN-Loaded Microemulsions. In order to find out the concentration range of components for the existing range of MEs, pseudoternary phase diagrams were constructed using water titration method at ambient temperature. Three phase diagrams were prepared with the $1: 1,2: 1$, and $3: 1$ weight ratios of caprylocaproyl macrogol8-glyceride to polyglyceryl oleate, respectively. For each phase diagram at a specific surfactant (S)/cosurfactant (CoS) mixing ratio $(\mathrm{Km})$, the ratios of oil to the mixture of $\mathrm{S} / \mathrm{CoS}$ were varied as $0.5: 9.5,1: 9,1.5: 8.5,2: 8,2.5: 7.5$, $3: 7,3.5: 6.5,4: 6,4.5: 5.5,5: 5,5.5: 4.5,6: 4,6.5: 3.5,7: 3$, $7.5: 2.5,8: 2,8.5: 1.5,9: 1$, and $9.5: 0.5$. The mixtures of oil and $\mathrm{S} / \mathrm{CoS}$ at certain weight ratios were diluted with water dropwise, under moderate magnetic stirring. After being equilibrated at ambient temperature for 24 hours, the mixtures were assessed visually and determined as being MEs, crude emulsions, or ME gels. The stable MEs were also observed under polarizing light to conform their isotropic nature. No attempt was made to distinguish between oilin-water, water-in-oil, or bicontinuous type MEs. Gels were claimed for those clear and highly viscous mixtures that did not show a change in the meniscus after being tilted to an angle of $90^{\circ}$.

ME formulation was selected from the pseudoternary phase diagram with $3: 1$ weight ratio of caprylocaproyl macrogol-8-glyceride to polyglyceryl oleate. ITN was added to the mixtures of oil and S/CoS, and then an appropriate amount of distilled water was added to the mixture drop by drop and the ME containing ITN was obtained by stirring the mixtures at ambient temperature which was stored at ambient temperature. Table 1 shows composition of selected ME formulation. 
TABle 1: Composition of selected microemulsions (\%w/w).

\begin{tabular}{lc}
\hline Components & Content \\
\hline Isotretinoin & $0.5 \% \mathrm{w} / \mathrm{w}$ \\
Isopropyl myristate & $4.0 \% \mathrm{wt} / \mathrm{wt}$ \\
Caprylocaproyl macrogol-8-glyceride & $31.5 \% \mathrm{wt} / \mathrm{wt}$ \\
Polyglyceryl oleate & $10.5 \% \mathrm{wt} / \mathrm{wt}$ \\
Aqueous phase & q. s. for $100 \% \mathrm{wt} / \mathrm{wt}$ \\
\hline
\end{tabular}

2.6. Characterization of ITN-Loaded Microemulsion. The average droplet size and polydispersity index of ME was measured by photon correlation spectroscopy (PCS) with in-built Zetasizer (Nano ZS, Malvern Instruments, UK) at $633 \mathrm{~nm}$. Helium neon gas laser having intensity of $4 \mathrm{~mW}$, was the light source. The droplet size was calculated using Stokes-Einstein relationship by Zetasizer Software. Transmission electron microscopy (TEM) was used to characterize the microstructure of ITN loaded ME. ME was placed on a carbon-coated copper grid and then a drop of $1 \%$ phosphotungstic acid covered on MEs. The superfluous phosphotungstic acid on MEs was wiped off by filter paper. The TEM images were obtained using a Tecnai G2 20 TEM (Philips, Holland). The refractive index of the system was measured by an Abbe refractometer (Bausch and Lomb Optical Company, Rochester, NY) by placing 1 drop of solution on the slide. The percent transmittance of the system was measured using a colorimeter (Digital Colorimeter, D-801, Photocon) at $570-590 \mathrm{~nm}$. In order to verify the isotropic nature of ME, samples were examined using cross-polarized light microscopy (Polarizing Microscope, Carlzeless, Jena, Germany). A drop of sample was placed between a cover slip and a glass slide and then observed under cross-polarized light. The $\mathrm{pH}$ values of ME was determined using digital $\mathrm{pH}$ meter (Orion pH meter 420A, Allometric Ltd., Baton Rouge, LA), standardized using $\mathrm{pH} 4$ and 7 buffers before use. The viscosity of ME was measured using a Brookfield Viscometer (Brookfield Engineering LABS, Stoughton, MA) with spindle LV-III at $100 \mathrm{rpm}$ using interval of 30 seconds. All aspects of testing were controlled using optional Rheocalc Software. The electric conductivity of ME was measured with a conductivity meter (Equip-Tronics, EQ-664, Mumbai, India) equipped with in-built magnetic stirrer. This was done by using conductivity cell (with a cell constant of 1.0) consisting of two platinum plates separated by desired distance and having liquid between the platinum plate acting as a conductor $[12,13]$.

2.7. Photostability Study of ITN in Solution. The photostability of ITN [24, 25] was assessed by recording its absorption spectra over the wavelength range of $200-500 \mathrm{~nm}$ in two matched quartz cells with a $1 \mathrm{~cm}$ light path using a double beam UV-Visible spectrophotometer (Perkin Elmer, Lambda 19, Norwalk, CT) at the following conditions: scan speed-slow; time response $1 \mathrm{~s}$; spectral band $1 \mathrm{~nm}$. The radiant power was adjusted to the lower value in the instrumental scale, and the cabinet temperature at $25^{\circ} \mathrm{C}$. These gentle experimental conditions were set because of the high sensitivity of the drug to light, allowing so to obtain

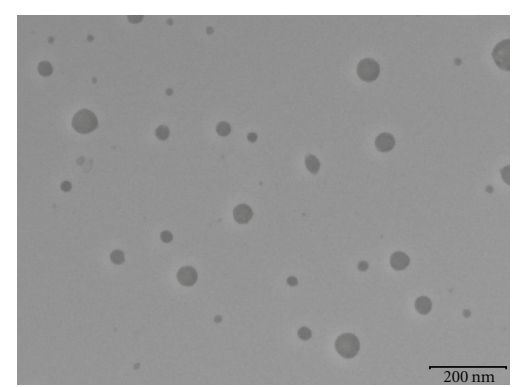

FIGURE 1: The TEM image of isotretinoin loaded microemulsion containing isopropyl myristate as an oil phase.

more accurate control of the photodegradation process. Methanolic solution of ITN (concentration: $20 \mu \mathrm{g} / \mathrm{mL}$ ) was exposed to natural sunlight ( $>20,000 \mathrm{Lux})$ and the UV spectra of the samples were recorded just after preparation $(t=0)$ and at the following time intervals: $15,30,60,90,120$, $150,180,210,240 \mathrm{~min}$, after suitable dilution with methanol. All sample solutions were filtered through nylon $0.45 \mu \mathrm{m}$ membrane filters.

2.8. Phototstability Study of ITN in Microemulsion. ME formulation was exposed to light under the same experimental conditions described above for solution, and recording the spectra at the same irradiation times. For ME formulation, spectrophotometric measurement was performed by diluting it suitably with methanol. Baseline correction was done using a plain ME dispersion diluted suitably with methanol to nullify any possible absorption arising from the excipients. All sample solutions were filtered through nylon $0.45 \mu \mathrm{m}$ membrane filters.

Sufficient care was taken to maintain similar experimental conditions for both the samples, that is, ITN in methanol and ITN in ME. Consequently, an interpretation of the photodegradation process of ITN by determination of kinetic parameters was tried as a complementary aim of the photostability study.

\section{Results and Discussion}

Particle size of plain ME and drug-loaded ME were determined, and there was no significant difference observed in average particle size after loading the drug. The ME formulation had the lowest average particle size $45 \pm 0.5 \mathrm{~nm}$ with polydispersity index (PI) of $0.145 \pm 0.027$. PI is a measure of particle homogeneity and it varies from 0.0 to 1.0 . The closer to zero the PI value the more homogenous are the particles. The PI showed that ME formulation had narrow size distribution. The TEM imaging of ITN loaded MEs is shown in Figure 1. The particle size of ITN loaded MEs from TEM images (Figure 1) accords with that of from PCS. The imaging showed that ITN loaded ME exhibited a spherical shape and had a narrow size distribution. The refractive index of the developed ME was found to be 1.329 and percent transmittance $>99 \%$ which proved the transparency of the system. The sample was examined by ocular inspection 


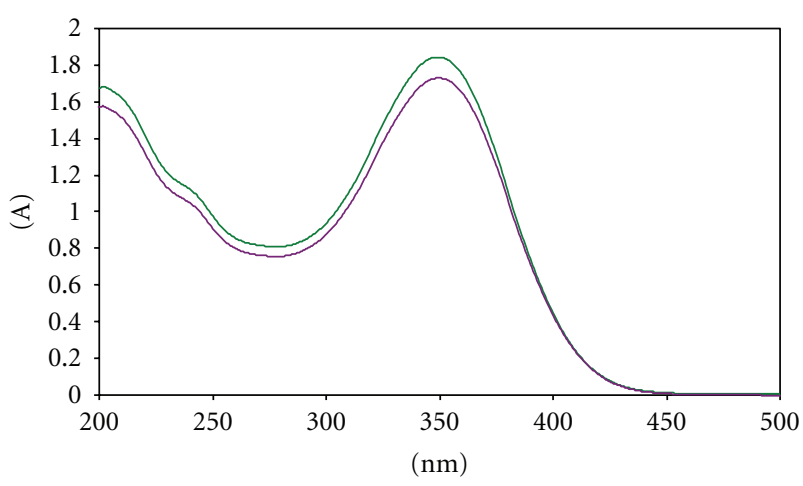

Figure 2: Overlaid UV spectra of $20 \mu \mathrm{g} / \mathrm{mL}$ ITN in methanol solution and $25 \mu \mathrm{g} / \mathrm{mL}$ ITN in microemulsion.

TABLE 2: Regression analysis of calibration curve for isotretinoin (ITN) by the proposed UV spectrophotometric method $(n=5)$.

\begin{tabular}{lc}
\hline Parameters & ITN \\
\hline Concentration range & $5-40 \mu \mathrm{g} / \mathrm{mL}$ \\
Slope & 0.1115 \\
Standard deviation of the slope & $0.57 \times 10^{-4}$ \\
Intercept & -0.1045 \\
Standard deviation of the intercept & $0.48 \times 10^{-3}$ \\
Correlation coefficient & 0.9998 \\
\hline
\end{tabular}

in a cross polarizer for sample homogeneity and birefringence. The ME appeared completely dark when observed under cross polarizer which indicated that it was optically isotropic. The ME formulation had appropriate observed $\mathrm{pH}$ value $(5.9 \pm 0.24)$ for topical application. Incorporation of ITN did not significantly affect the observed $\mathrm{pH}$ value of the ME formulations. The developed system had the low viscosity $(31.54 \pm 0.23 \mathrm{mPa} \cdot \mathrm{s})$ and high conductivity $(149.5 \pm 2.21 \mu \mathrm{S} / \mathrm{cm})$. There was no significant difference found between the viscosities of plain and drug-loaded MEs. The investigated ME formulation containing nonionic surfactant mixture, oil, and water showed electroconductive behaviour in spite of its non-ionic nature. From the viscosity and electroconductive study it could be concluded the prepared ME formulation was of o/w type $[12,13]$.

3.1. UV Spectra of ITN. Figure 2 shows the UV spectra of ITN in methanol solutions. As evident, ITN is characterized by the presence of one imposing absorbance maximum at $348 \mathrm{~nm}$. Calibration graphs were obtained by applying least squares regression analysis to the absorbance amplitudes at the single-maximum peaks against the increasing concentrations of pure ITN. The calibration curves parameters are summarized in Table 2.

3.2. Photostability of ITN in Solution. The spectral curves recorded on the methanolic solution of ITN at the various times of light exposure are shown in Figure 3. The light exposure caused a sharp degradation after 15 minutes of irradiation with a contemporary shift of maximum peak

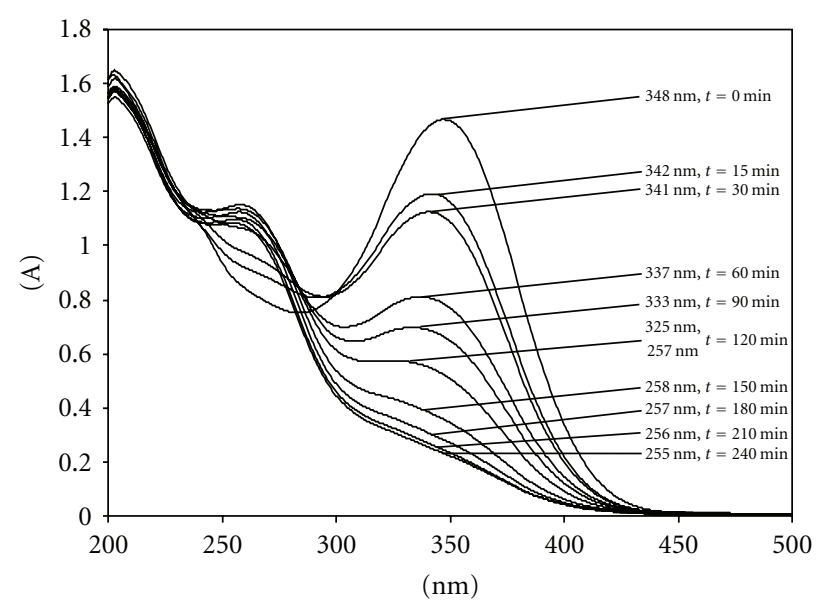

FIGURE 3: UV spectra of a $20 \mu \mathrm{g} / \mathrm{mL}$ ITN in methanol solution after different times of light exposure.

from 348 to $342 \mathrm{~nm}$ indicating the isomerization of ITN to 9cis isomer [25]. A slower degradation was observed later on with the minimization of the $337 \mathrm{~nm}$ maximum peak with a 50\% absorbance lowering, compared to the initial value. Further light exposure caused the minimization of this peak with its simultaneous shift to lower wavelengths, probably due to a complex mixture of several retinoic acid isomers. Such results point out the very high sensitivity to light of ITN, suggesting the importance of the development of a pharmaceutical carrier able to minimize its photodegradation. Recent studies have demonstrated the positive action of the novel formulation matrix as carrier systems for a number of photosensitive drugs [19-25]. The first goal of the present study was to investigate the photostabilization of the ITN when loaded in ME through data obtained under conditions of accelerated irradiation. Consequently, an interpretation of the photodegradation process of ITN drug was tried as a complementary study.

3.3. Photostability of ITN in Microemulsion. The photostability studies were carried out by spectrophotometric measurements performed on the ITN loaded ME formulation just before exposure and exposure at increasing times by diluting the samples suitably with methanol. Figure 4 shows the spectral curves of the ITN loaded ME formulation obtained after the sequential exposure times. As can be seen, the ITN in ME carrier, strongly reduced the photodegradation process as compared to that of the methanolic solution. On the other hand, the photostability of the ITN in ME formulation improved as compared to that recorded of the methanolic solution. Furthermore, significant shift of the maximum peak at $348 \mathrm{~nm}$ to lower wavelength was not observed. This may be considered as an interesting result, as it demonstrates no direct isomerization of ITN to 9-cis isomers [25]. Also, a residual ITN concentration of $75 \%$ was still present after irradiation for and $240 \mathrm{~min}$ which was significantly higher in comparison to ITN concentration in methanolic solution at the end of $240 \mathrm{~min}(P<0.05)$. The dramatic improvement in the photostability of ITN indicates positive action of the ME matrix as a carrier system. 


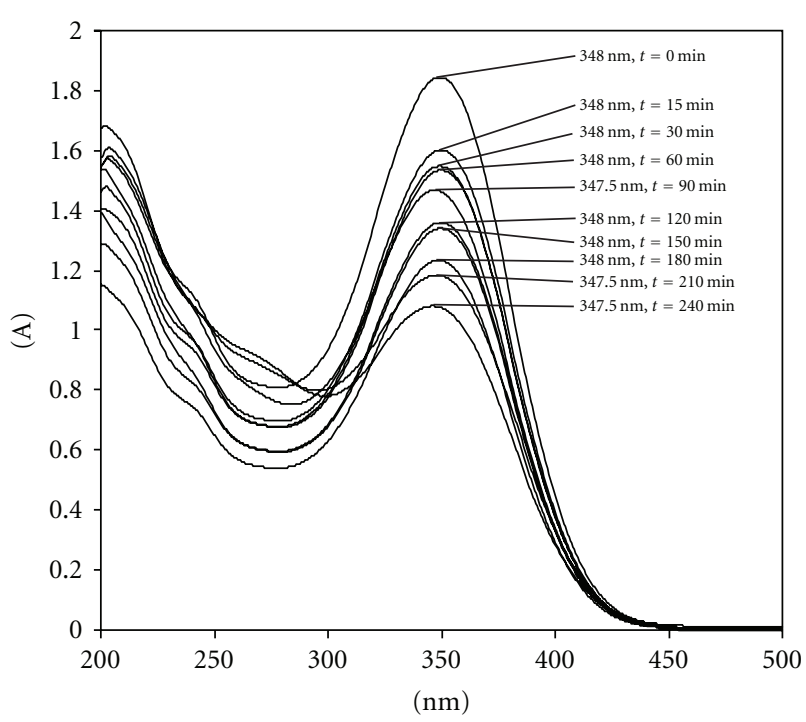

FIGURE 4: UV spectra of a $25 \mu \mathrm{g} / \mathrm{mL}$ ITN in microemulsion after different times of light exposure.

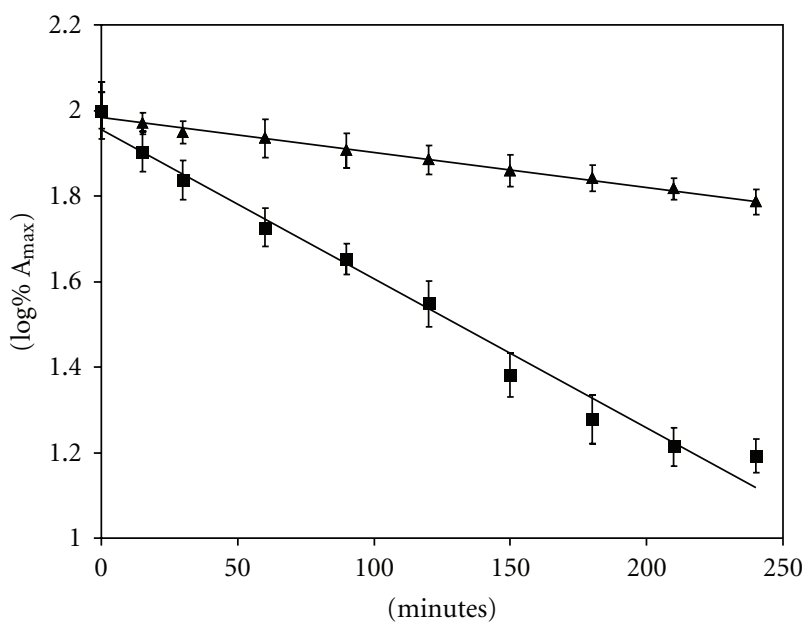

FIGURE 5: Photodegradation plots of ITN solution in methanol and microemulsion containing $\operatorname{ITN}(\boldsymbol{\Delta})$. All values are means of three replicated experiments.

In order to evaluate photostability of ME excipients, a parallel analytical control on the formulation matrix photostability was also performed. For this purpose, sample of plain ME was irradiated under the same experimental conditions adopted for the ITN loaded ME. No appreciable changes between the spectra recorded before and after light exposure were detected, clearly showing that any photodegradation occurring during experimental time was negligible.

Ioele et al. reported that the inclusion of ITN in liposomes did not give satisfactory results and shows higher degradation rate than the retinoic acid in liposomes complex studied, but showing an increased stability if compared with the ITN in ethanol solution and concluded that ITN in liposomes showed a worse performance among the drugs
TABLE 3: Rate constants* of photodegradation for ITN in methanol solution and microemulsion $(n=3)$.

\begin{tabular}{lcccc}
\hline Drug & Sample matrix & $K$ & $t_{0.5}$ & $R^{2}$ \\
\hline \multirow{2}{*}{ ITN } & Methanol solution & $8.06 \times 10^{-3}$ & 86 & 0.9830 \\
& Microemulsion & $1.84 \times 10^{-3}$ & 377 & 0.9899 \\
& formulation & & & \\
\hline
\end{tabular}

${ }^{*} t$ is expressed in minutes.

studied, probably due to a low inclusion as a result of nonlinear molecular structure [25]. In current study, a residual ITN concentration of $75 \%$ was found to be present after irradiation for $240 \mathrm{~min}$ in ME. On the contrary, in previous study [25] the inclusion of ITN in liposomes did not give analogous satisfactory results, residual ITN concentration was less than $65 \%$.

In a nutshell, we can conclude that ME exhibits a good potential to improve the photostability of ITN and can be employed as a valuable drug delivery strategy.

3.4. Kinetic Parameters Determination. The photochemical reaction in methanol solution was demonstrated to cause a very rapid isomerization of ITN followed by a further degradation consisting in the minimization of the absorbance peaks. Therefore, the absorbance values of these maxima were used to evaluate the kinetics of the photodegradation processes [25]. ITN was shown to follow a first order kinetics and a good linearity was obtained by plotting the logarithm of absorbances as a function of time, both in methanolic solution and in ME, in accordance with the following equation:

$$
\log (\% \mathrm{~A})=-k t+2
$$

where, $\% A$ was percent residual absorbance, $k$ was the photodegradation rate constant, $t$ was the time $(\min )$, and 2 was the logarithm of initial percent absorbance (100\%).

The degradation was evaluated on the basis of kinetic photodegradation constant $k$ and half-life time $\left(t_{0.5}\right)$, with respect to the initial percent absorbance. ITN degradation curves are plotted in Figure 5 while Table 3 summarizes the degradation kinetic parameters based on data from three replicate analyses for each sample. ME demonstrated to increase ITN half-life about five-times in comparison with a methanol solution, under a direct sun light.

\section{Conclusion}

As it is evident, the ITN inclusion in ME results in a good photostability of the drug, whereas in methanol ITN degraded almost completely after about 240 min under a direct sun light, while in the ME matrix still $75 \%$ of residual ITN concentration could be measured. Results obtained from this photostability study lead us to suppose that the better photoprotection of ITN in ME matrix is the consequence of the inclusion of this drug in ME matrix. Further, studied kinetics of the photodegradation processes for ITN loaded ME formulation demonstrated to increase 
ITN half-life about five-times in comparison with a methanol solution, under a direct sun light.

The ME system described in the present paper should provide a valuable tool for the development of new pharmaceutical formulations of ITN, capable of improving their photostability.

\section{Acknowledgments}

The authors are thankful to Astron Research Ltd. for the gift sample of ITN pure powder, Sophisticated Instrumentation Center for Applied Research and Testing (SICART; Vallabh Vidyanagar, India) for providing facilities for carrying out analytical work, and Gattefosse (Saint-Priest, France) for providing gratis samples of excipients.

\section{References}

[1] S. Feldman, R. E. Careccia, K. L. Barham, and J. Hancox, "Diagnosis and treatment of acne," American Family Physician, vol. 69, no. 9, pp. 2123-2130, 2004.

[2] J. J. Leyden, "Topical treatment of acne vulgaris: retinoids and cutaneous irritation," Journal of the American Academy of Dermatology, vol. 38, no. 4, pp. S1-S4, 1998.

[3] A. L. Zaenglein, "Topical retinoids in the treatment of acne vulgaris," Seminars in Cutaneous Medicine and Surgery, vol. 27, no. 3, pp. 177-182, 2008.

[4] J. G. Allen and D. P. Bloxham, "The pharmacology and pharmacokinetics of the retinoids," Pharmacology \& Therapeutics, vol. 40, no. 1, pp. 1-27, 1989.

[5] S. Kang, G. G. Krueger, E. A. Tanghetti et al., "A multicenter, randomized, double-blind trial of tazarotene $0.1 \%$ cream in the treatment of photodamage," Journal of the American Academy of Dermatology, vol. 52, no. 2, pp. 268-274, 2005.

[6] R. W. Lucek and W. A. Colburn, "Clinical pharmacokinetics of the retinoids," Clinical Pharmacokinetics, vol. 10, no. 1, pp. 38-62, 1985.

[7] C. C. Zouboulis, "Retinoids-which dermatological indications will benefit in the near future?" Skin Pharmacology and Applied Skin Physiology, vol. 14, no. 5, pp. 303-315, 2001.

[8] L. Boltri, S. Morel, M. Trotta, and M. R. Gasco, "In vitro transdermal permeation of nifedipine from thickened microemulsions," Journal de Pharmacie de Belgique, vol. 49, no. 4, pp. 315-320, 1994.

[9] F. P. Bonina, V. Carelli, G. Di Colo, L. Montenegro, and E. Nannipieri, "Vehicle effects on in vitro skin permeation of and stratum corneum affinity for model drugs caffeine and testosterone," International Journal of Pharmaceutics, vol. 100, no. 1-3, pp. 41-47, 1993.

[10] M. B. Delgado-Charro, G. Iglesias-Vilas, J. Blanco-Mendez, M. A. López-Quintela, J. P. Marty, and R. H. Guy, "Delivery of a hydrophilic solute through the skin from novel microemulsion systems," European Journal of Pharmaceutics \& Biopharmaceutics, vol. 43, no. 1, pp. 37-42, 1997.

[11] M. Kreilgaard, E. J. Pedersen, and J. W. Jaroszewski, "NMR characterisation and transdermal drug delivery potential of microemulsion systems," Journal of Controlled Release, vol. 69, no. 3, pp. 421-433, 2000.

[12] M. R. Patel, R. B. Patel, J. R. Parikh, A. B. Solanki, and B. G. Patel, "Effect of formulation components on the in vitro permeation of microemulsion drug delivery system of fluconazole," AAPS PharmSciTech, vol. 10, no. 3, pp. 917-923, 2009.
[13] M. R. Patel, R. B. Patel, J. R. Parikh, A. B. Solanki, and B. G. Patel, "Investigating effect of microemulsion components: in vitro permeation of ketoconazole," Pharmaceutical Development \& Technology, vol. 16, no. 3, pp. 250-258, 2011.

[14] ICH harmonized tripartite guideline, "Photostability testing of new drug substance and products," Federal Register, vol. 62, no. 95, pp. 27115-27122, 1997.

[15] N. H. Anderson, D. Johnston, M. A. Mclelland, and P. Munden, "Photostability testing of drug substances and drug products in UK pharmaceutical laboratories," Journal of Pharmaceutical \& Biomedical Analysis, vol. 9, no. 6, pp. 443449, 1991.

[16] W. Aman and K. Thoma, "The influence of formulation and manufacturing process on the photostability of tablets," International Journal of Pharmaceutics, vol. 243, no. 1-2, pp. 33-41, 2002.

[17] H. H. Tønnesen, "Formulation and stability testing of photolabile drugs," International Journal of Pharmaceutics, vol. 225, no. 1-2, pp. 1-14, 2001.

[18] M. G. Brisaert, I. Everaerts, and J. A. Plaizier-Vercammen, "Chemical stability of tretinoin in dermatological preparations," Pharmaceutica Acta Helvetiae, vol. 70, no. 2, pp. 161166, 1995.

[19] L. H. Wang, "Simultaneous determination of retinal, retinol and retinoic acid (all-trans and 13-cis ) in cosmetics and pharmaceuticals at electrodeposited metal electrodes," Analytica Chimica Acta, vol. 415, no. 1-2, pp. 193-200, 2000.

[20] G. Ragno, M. Veronico, R. Maddalena, and C. Vetuschi, "Tretinoin assay in cosmetics and pharmaceuticals by carbon phase extraction," Journal of the Society of Cosmetic Chemists, vol. 47, no. 5, pp. 325-336, 1996.

[21] K. Thoma and U. E. Joachan, "Liposome dermatics: assessment of long-term stability," in Liposome Dermatics, Griesbach Conference, O. Braun-Falco, H. C. Korting, and H. I. Maibach, Eds., pp. 150-156, Springer, Berlin, Germany, 1992.

[22] M. Brisaert, M. Gabriëls, V. Matthijs, and J. PlaizierVercammen, "Liposomes with tretinoin: a physical and chemical evaluation," Journal of Pharmaceutical and Biomedical Analysis, vol. 26, no. 5-6, pp. 909-917, 2001.

[23] M. Manconi, C. Sinico, D. Valenti, G. Loy, and A. M. Fadda, "Niosomes as carriers for tretinoin. I. Preparation and properties," International Journal of Pharmaceutics, vol. 234, no. 1-2, pp. 237-248, 2002.

[24] K. A. Shah, A. A. Date, M. D. Joshi, and V. B. Patravale, "Solid lipid nanoparticles (SLN) of tretinoin: potential in topical delivery," International Journal of Pharmaceutics, vol. 345, no. 1-2, pp. 163-171, 2007.

[25] G. Ioele, E. Cione, A. Risoli, G. Genchi, and G. Ragno, "Accelerated photostability study of tretinoin and isotretinoin in liposome formulations," The International Journal of Pharmaceutics, vol. 293, no. 1-2, pp. 251-260, 2005. 

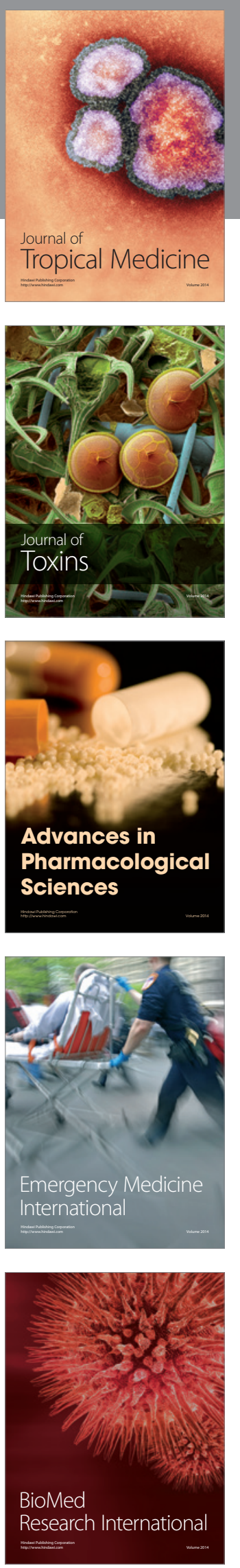
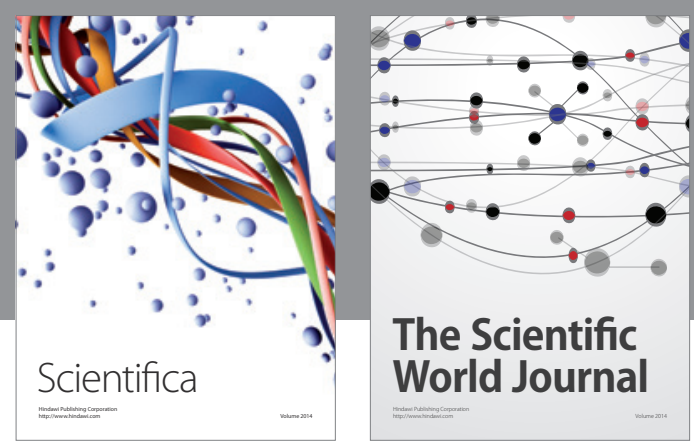

The Scientific World Journal
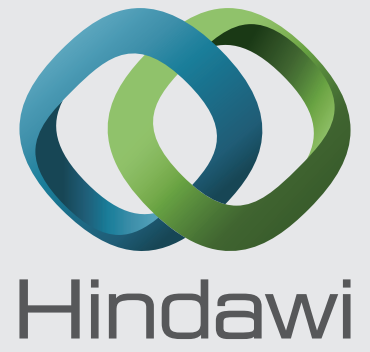

Submit your manuscripts at

http://www.hindawi.com
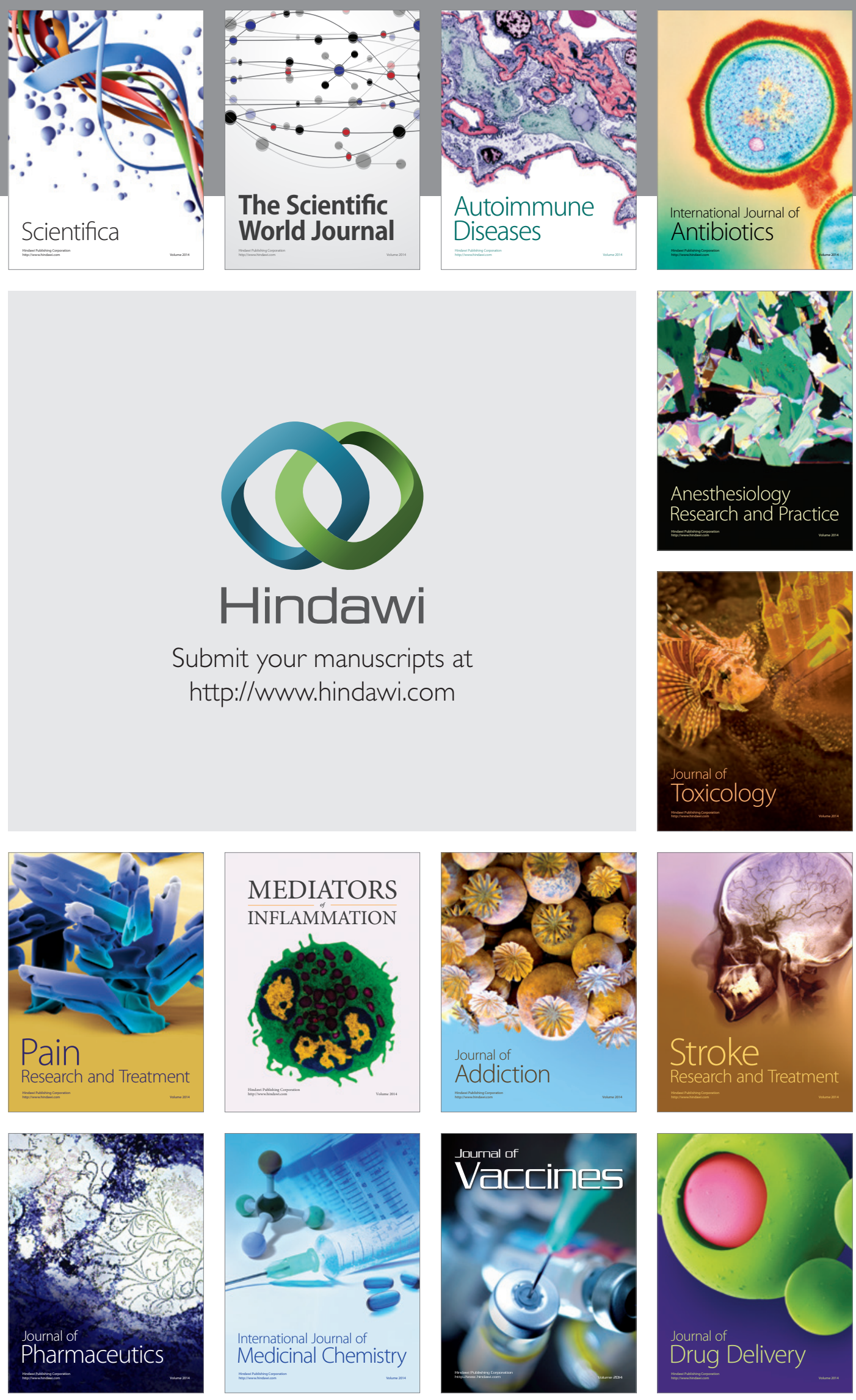